\title{
The impact of using calibrated utensils to assess portion sizes in infants aged 4-18 months
}

\author{
J. Delve ${ }^{1}$, S. West-Sadler ${ }^{2}$, E. Foster ${ }^{1}$, J. Sommerville ${ }^{2}$, R. Stratton ${ }^{3}$, A. M. Stephen ${ }^{2}$ and A. Adamson ${ }^{1}$ \\ ${ }^{1}$ Institute of Health \& Society, Human Nutrition Research Centre, Newcastle University, Framlington Place, \\ Newcastle upon Tyne NE2 4HH, ${ }^{2}$ MRC Human Nutrition Research, Elsie Widdowson Laboratory, Fulbourn Road, \\ Cambridge CB1 9NL and ${ }^{3}$ Department of Health, Wellington House, 133-155 Waterloo Road, London SE1 8UG, UK
}

Fieldwork for the Diet and Nutrition Survey of Infants and Young Children (DNSIYC) was carried out during 2011 to provide detailed quantitative information on food and nutrient intakes, nutrient sources and nutritional status of a representative sample of infants aged 4-18 months in the UK. In 2009-10 a preliminary study was carried out to assess the effectiveness and validity of using calibrated utensils to measure food and drink consumption in infants aged 4-18 months. The aim was to determine if the use of calibrated utensils influenced the portion size offered to the infant and so affect their daily nutrient intake.

Food and drink intakes of 50 infants in North-East England were recorded using both an ESTIMATED and a WEIGHED 4-day food diary in random order, completed by the infant's parent/carer, over a 2 week period. Parents were provided with 4 graduated containers for food preparation and serving $(2 \times 150 \mathrm{ml}$ and $2 \times 300 \mathrm{ml})$, as well as 6 spoons $(1.25 \mathrm{ml}-15 \mathrm{ml})$, and were encouraged to use these to record all portion sizes in the ESTIMATED diary. Volume measures were converted to gram weights by the application of conversion factors, calculated for each food and drink consumed.

The table below shows the mean daily intakes for each method. $41 \%$ of the sample had mean daily intakes $(\mathrm{g})$ for the estimated method which were within $10 \%$ of the weighed intakes. $96 \%$ were within $50 \%$ of the weighed intakes. There was no difference in mean intakes between the two methods for different food types e.g. discrete food items and amorphous foods.

\begin{tabular}{lcc}
\hline Mean daily intakes & Weighed & Estimated \\
\hline Food weight $(\mathrm{g})$ & 932 & 958 \\
Energy $(\mathrm{kcal})$ & 721 & 776 \\
\hline
\end{tabular}

The results indicated that the estimated intake method, supported by the use of measuring equipment, gave very similar results to those of weighed intakes. However the diary coding stage for these pilot data found the use of the equipment resulted in a much slower coding rate and a high number of queries. Based on the number of participants taking part in the main stage of DNSIYC, it was calculated many more coders would be required to manage the queries and meet deadlines. The final decision was to proceed with a household measures approach for dietary data collection; this method required no additional equipment and fewer coding resources.

Although the household measures method has been extensively used in older age groups, ${ }^{(1)}$ further work is proposed to validate this method against weighed intakes in 4-18 month infants. The study will follow the same method described above and will determine whether the household measures approach results in similar intakes as the weighed method.

1. Bates B, Lennox A, Bates C, Swan G (2011) National Diet and Nutrition Survey- Headline results from years 1 and 2 (combined) of the Rolling Programme (2008/9-09/10). Department of Health. 